\title{
Exercise Echocardiography in Asymptomatic or Minimally Symptomatic Chronic Severe Aortic Regurgitation
}

\author{
- A New Era in the Search for 'the Golden Moment' for Surgery -
}

\author{
Kyung-Hee Kim, MD; Hyung-Kwan Kim, MD
}

$\mathbf{S}$ evere aortic regurgitation (AR) gradually results in irreversible left ventricular (LV) dysfunction, heart failure, and death. Aortic valve replacement (AVR) can minimize or abolish AR symptoms and should improve prognosis. Because studies of the natural history of AR have suggested that symptomatic patients have an excessive mortality rate as compared with asymptomatic patients and that the severity of preoperative symptoms is a strong determinant of survival after AVR, ${ }^{1}$ symptom onset represents a most important surgical indication in chronic severe $\mathrm{AR}^{2}{ }^{2}$ In this respect, it seems intuitively reasonable that AVR should be performed before symptoms begin and before any changes leading to irreversible LV dysfunction are impending. But how can we identify this "golden" time point for AVR? Apart from symptom onset, sudden death is a real threat for asymptomatic patients with subclinical myocardial dysfunction, even if those patients are found to have a normal $\mathrm{LV}$ ejection fraction (LVEF). ${ }^{3}$ Therefore, it is as clear as daylight that we should need indexes that best reflect the myocardial function in chronic severe AR.

\section{Article p 2390}

As with aortic stenosis, ${ }^{4}$ it has been anticipated that exercise echocardiography (ExEcho) may unmask symptoms in patients with severe AR who are otherwise asymptomatic or minimally symptomatic, finally identifying surgical candidates before LV irreversible dysfunction occurs. ${ }^{3}$ Specifically, in patients with normal function at rest, an exercise-induced increase in LVEF pre-surgery, or positive contractile reserve $(\mathrm{CR}(+))$, predicts improvement in postoperative LV function. ${ }^{3}$ However, ExEcho is not currently recommended for this purpose. According to the ACC/AHA guideline, the main indication for ExEcho in $\mathrm{AR}$ is to elicit symptoms in patients with a "subjective" exercise limitation or before participation in athletic activity. ${ }^{3}$ The ESC guideline does "NOT" recommend ExEcho for routine clinical use, mainly based on the sparse data supporting its incremental prognostic value. ${ }^{5}$

In this issue of the Journal, Park et al investigate CR in asymptomatic or minimally symptomatic patients with chronic severe AR using treadmill ExEcho. ${ }^{6}$ They studied 58 minimally symptomatic patients with chronic severe AR whose diagnosis was based on comprehensive echocardiographic evaluation. ${ }^{7}$
The patients underwent symptom-limited treadmill ExEcho using a protocol selected according to individual's cardiovascular and overall physical status. The authors found that although CR related best to the baseline LV end-systolic dimension (LVESD) index, a significant proportion of patients had discordance between the presence or absence of CR and the $\mathrm{LV}$ dimensions at rest. Given that the resting $\mathrm{LV}$ dimension is an important criterion determining optimal AVR timing, Park et al conclude that ExEcho could further identify patients who would not qualify for AVR based on the current guidelines, ${ }^{2,5}$ but who have subclinical myocardial dysfunction and could benefit from AVR. In the evaluation of valvular disease, physiologic exercise is usually adopted as the stressor rather than pharmacologic challenge. ExEcho allows comprehensive evaluation of each patient, including valve status itself, functional capacity of the patient, and exercise-induced changes in symptoms or LV function. ${ }^{8}$ Notwithstanding, it is hitherto unknown which protocol (supine bicycle vs. treadmill) can provide clearer prognostic information. Treadmill ExEcho is physiologically better, although image acquisition can be performed at every stage through the bicycle protocol. Additionally, because interpretation is based on a comparison of resting and peak exercise images, treadmill ExEcho seems to be more preferable. However, no solid evidence is yet available.

Myocardial contractility can be assessed by the end-systolic pressure-volume relationship independent of LV preload and afterload. ${ }^{9}$ As myocardial contractility decreases, the slope of the relationship declines and moves to the right. ${ }^{9}$ However, the change in this relationship is difficult to detect clinically and thus we expect that changes in LVEF and/or LV dimension follow more linearly than in the end-systolic pressure-volume relationship, which is the main reason why the LVEF and LV dimension are included in the current guidelines. ${ }^{2,5}$ However, this is not always true, and Park et al have provided new evidence. Although $\mathrm{CR}(+)$ is mainly determined by the LVESD index, they observed that one-third of the patients with $\mathrm{CR}(+)$ had a LVESD $>55 \mathrm{~mm}$, and, more importantly, a similar proportion of patients with $\mathrm{CR}(-)$ had a relatively smaller LVESD. Therefore, we can presume that current indexes used for determining surgical timing in cases of minimally symptomatic severe AR are limited, and ExEcho could provide useful information additional to LVEF and LV dimension available with

The opinions expressed in this article are not necessarily those of the editors or of the Japanese Circulation Society.

Received July 8, 2013; accepted July 9, 2013; released online July 26, 2013

Department of Internal Medicine, Seoul National University College of Medicine, Cardiovascular Center, Seoul National University Hospital, Seoul, Korea

Mailing address: Hyung-Kwan Kim, MD, PhD, Associate Professor, Division of Cardiology, Department of Internal Medicine, Seoul

National University College of Medicine, 101 Daehak-ro, Jongno-gu, Seoul 110-744, Korea. E-mail: hkkim73@ snu.ac.kr

ISSN-1346-9843 doi:10.1253/circj.CJ-13-0874

All rights are reserved to the Japanese Circulation Society. For permissions, please e-mail: cj@j-circ.or.jp 


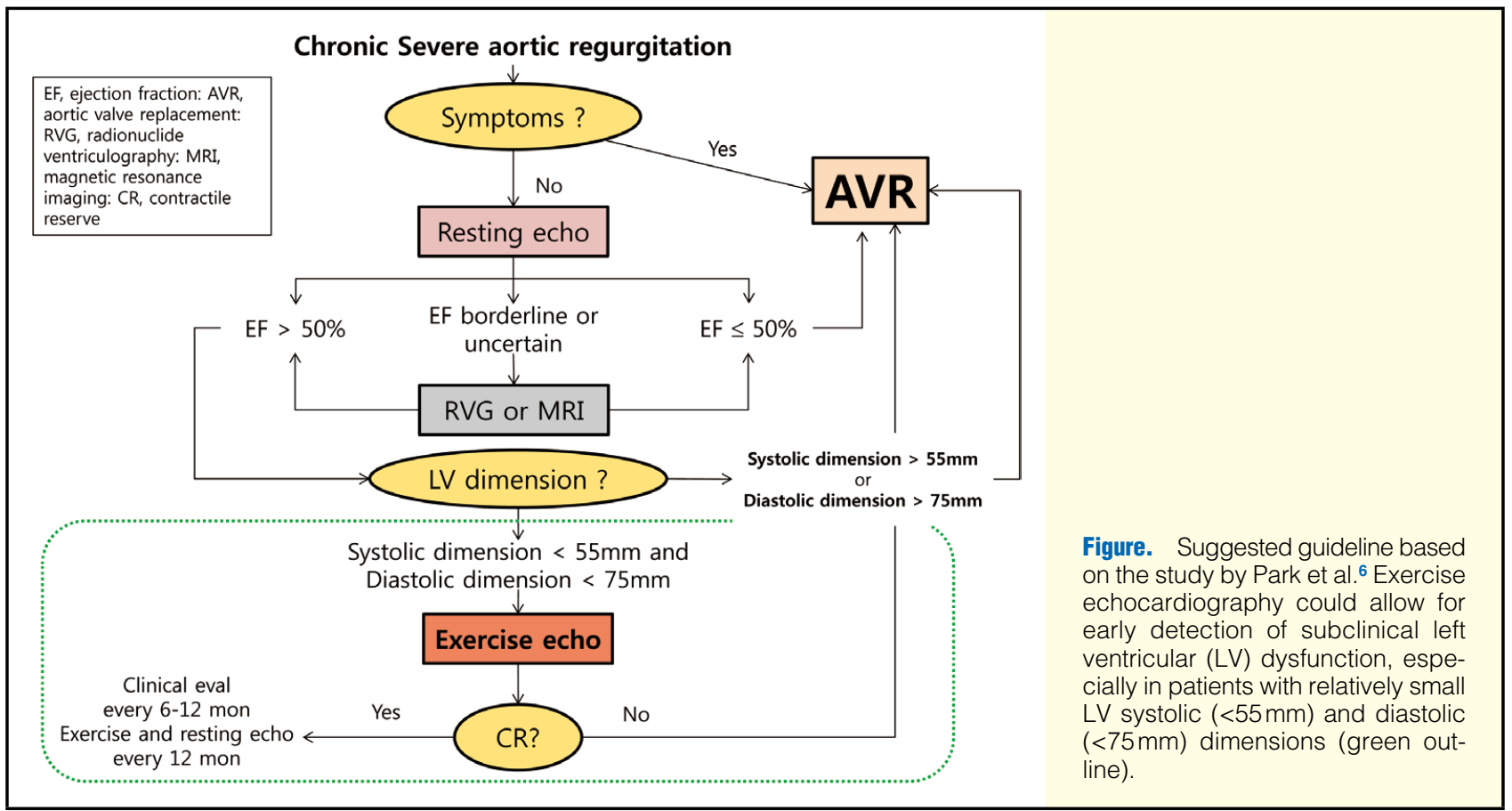

resting echocardiography. If confirmed in future studies, the current guidelines could be simplified as shown in the Figure.

Although these authors are to be congratulated for this nicely executed study, several limitations should be addressed. First, they defined $\mathrm{CR}(+)$ as LVEF increment after exercise, without considering the degree of LVEF increment. LVEF can vary widely, even in an individual patient, ${ }^{10}$ the effect of which may be more pronounced in the immediate post-exercise measurement of LVEF. Second, there is a possibility that the change in LVEF after exercise is not influenced by myocardial contractility alone. In fact, Kawanishi et al demonstrated that the change in LVEF is directly related to the change in systemic vascular resistance. ${ }^{11}$ Third, a consensus has not been reached on which exercise modality is more appropriate or more predictable for future outcomes, although the treadmill exercise is more physiological. Head-to-head comparison data are clearly warranted. Lastly, most previous studies, including that by Park et al, only recruited a small number of AR patients., ${ }^{4,6,7,9}$ Larger, multicenter studies are needed to overcome this problem.

Although we cannot conclude that ExEcho should be used for asymptomatic or minimally symptomatic patients with severe AR, ${ }^{9}$ based only on the study by Park et al, it is high time to accept the potential usefulness of ExEcho in the decision-making process for optimal surgical timing. It could be a felicitous closing remark to state that a kick-off of larger prospective studies using ExEcho in patients with severe AR is strongly warranted.

\section{Disclosures}

None.

\section{References}

1. Dujardin KS, Enriquez-Sarano M, Schaff HV, Bailey KR, Seward JB, Tajik AJ. Mortality and morbidity of aortic regurgitation in clinical practice: A long-term follow-up study. Circulation 1999; 99: 18511857.

2. Bonow RO, Carabello BA, Chatterjee K, de Leon AC Jr, Faxon DP, Freed MD, et al. 2008 Focused update incorporated into the ACC/
AHA 2006 guidelines for the management of patients with valvular heart disease: A report of the American College of Cardiology/ American Heart Association Task Force on Practice Guidelines: Endorsed by the Society of Cardiovascular Anesthesiologists, Society for Cardiovascular Angiography and Interventions, and Society of Thoracic Surgeons. Circulation 2008; 118: e523-e661.

3. Borer JS, Hochreiter C, Herrold EM, Supino P, Aschermann M, Wencker D, et al. Prediction of indications for valve replacement among asymptomatic or minimally symptomatic patients with chronic aortic regurgitation and normal left ventricular performance. Circulation 1998; 97: 525-534.

4. Seo JS, Jang MK, Lee EY, Yun SC, Kim DH, Song JM, et al. Evaluation of left ventricular diastolic function after valve replacement in aortic stenosis using exercise Doppler echocardiography. Circ J 2012; 76: 2792-2798.

5. Vahanian A, Alfieri O, Andreotti F, Antunes MJ, Barón-Esquivias G, Baumgartner $\mathrm{H}$, et al. Guidelines on the management of valvular heart disease: Joint Task Force on the Management of Valvular Heart Disease of the European Society of Cardiology (ESC); European Association for Cardio-Thoracic Surgery (EACTS). Eur Heart J 2012; 33: $2451-2496$.

6. Park SJ, Enriquez-Sarano M, Song JE, Lee YJ, Ha MR, Chang SA, et al. Contractile reserve determined on exercise echocardiography in patients with severe aortic regurgitation. Circ J 2013; 77: 23902398.

7. Zoghbi WA, Enriquez-Sarano M, Foster E, Grayburn PA, Kraft CD, Levine RA, et al. Recommendations for evaluation of the severity of native valvular regurgitation with two-dimensional and Doppler echocardiography. J Am Soc Echocardiogr 2003; 16: 777-802.

8. Sicari R, Nihoyannopoulos P, Evangelista A, Kasprzak J, Lancellotti $\mathrm{P}$, Poldermans D, et al. Stress echocardiography expert consensus statement: European Association of Echocardiography (EAE) (a registered branch of the ESC). Eur J Echocardiogr 2008; 9: 415-437.

9. Suga H, Sagawa K. Instantaneous pressure-volume relationships and their ratio in the excised, supported canine left ventricle. Circ Res 1974; 35: 117-126.

10. Soliman OI, Kirschbaum SW, van Dalen BM, van der Zwaan HB, Mahdavian Delavary B, Vletter WB, et al. Accuracy and reproducibility of quantitation of left ventricular function by real-time threedimensional echocardiography versus cardiac magnetic resonance. Am J Cardiol 2008; 102: 778-783.

11. Kawanishi DT, McKay CR, Chandraratna PA, Nanna M, Reid CL, Elkayam U, et al. Cardiovascular response to dynamic exercise in patients with chronic symptomatic mild-to-moderate and severe aortic regurgitation. Circulation 1986; 73: 62-72. 